\title{
O Ensino de Semiologia Médica sob a Visão dos Alunos: Implicações para a Reforma Curricular
}

PALAVRAS-CHAVE:

-Educação Médica;

-Exame físico;

-Competência Clínica.

KEY-WORDS:

-Medical Education;

-Physical Examination;

-Clinical Competence.

Recebido em: 13/12/2005

Reencaminhado em: 04/04/2007

Aprovado em: 20/07/2007

$32 \mid \begin{aligned} & \text { REVISTA BRASILEIRA DE EDUCAÇÃO MÉDICA } \\ & 32(1): 32-38 ; 2008\end{aligned}$

\section{The Teaching of Clinical Examination from the View of the Students: Implications for a Curricular Reform}

Rose Mary Ferreira Lisboa da Silva ${ }^{1}$ Nilton Alves Rezende ${ }^{1}$

\begin{abstract}
RESUMO
As faculdades de Medicina devem acompanhar as mudanças na política de saúde e os desafios da assistência médica com novas propostas de ensino-aprendizagem, contemplando o projeto pedagógico centrado no aluno. Apesar disso, a visão discente raramente tem servido de parâmetro para essa reforma. Com este objetivo, foi aplicado em 2004 um questionário para todos os alunos do terceiro ano do curso de graduação de Medicina da Universidade Federal de Minas Gerais, sendo a participação voluntária e anônima. Esse questionário foi composto para se avaliar a importância do ciclo básico no ensino da Semiologia, os cenários de prática e o desempenho discente. Segundo os resultados, a Fisiologia foi considerada a mais importante do ciclo básico, seguida da Anatomia. A maioria dos alunos se considerou apta a fazer a anamnese, mas realizava o exame físico e o raciocínio fisiopatológico principalmente com ajuda docente. Quanto ao cenário de prática, houve uma preferência pelo ensino que englobasse a prática no ambulatório e na enfermaria. Concluiu-se que questionários específicos e apropriados podem ser úteis para um melhor planejamento pedagógico com a participação ativa dos alunos e para assegurar a integração horizontal e vertical das disciplinas.
\end{abstract}

\begin{abstract}
Medical Schools need to address the recent changes in the health policies and the challenges posed to the health care system with new recommendations for a curricular reform focused on the student. The viewpoint of the students and their performance however are rarely taken into account in the curricular decision-making. Therefore, in 2004, all third-year students of the graduate course of Medicine of the Federal University of Minas Gerais were invited to answer a questionnaire designed for analyzing their opinion about the importance of the disciplines of the first cycle in the teaching of medical semiology, their clinical skills and the best scenario for initiating the clinical practice. The participation in the study was voluntary and anonymous. According to the results physiology was considered the most important area influencing the clinical examination followed by anatomy. Most of the students considered themselves qualified for performing anamnesis. They also suggested a combination of inpatient and outpatient care as the best scenario for initiating their clinical practice. It is concluded that specific questionnai-
\end{abstract}

\footnotetext{
* Trabalho vencedor do Prêmio ABEM/2006 de Educação Médica, no $44^{\circ}$ Congresso Brasileiro de Educação Médica, realizado no período de 21 a 24 de setembro de 2006, em Gramado, Rio Grande do Sul, Brasil. (2º Lugar).

1 Universidade de São Paulo, São Paulo, Brasil.
} 
res, appropriately applied, would be useful both for a better pedagogical planning with active participation of the students and for ensuring horizontal and vertical integration of interdisciplinary components of the medical curriculum.

\section{INTRODUÇÃO}

O avanço da medicina e as mudanças na política de saúde têm implicações significativas para o currículo das Faculdades de Medicina, refletindo-se nas novas propostas de ensino-aprendizagem. Com o propósito de adequar esse ensino às atuais necessidades de assistência, o Conselho Nacional de Educação instituiu as Diretrizes Curriculares Nacionais do Curso de Graduação em Medicina, definindo as competências e habilidades gerais e específicas ${ }^{1}$. Esta preocupação em definir os requisitos globais essenciais com o objetivo de assegurar a excelência da qualidade dos serviços de saúde, associada ao respeito e ao bem-estar dos pacientes, é também um movimento de globalização, haja vista a recente criação do Instituto para a Educação Médica Internacional (Institute for International Medical Education - IIME)2,3.

Atualmente, em nosso país, ocorre uma discussão sobre a reforma curricular das faculdades de Medicina ${ }^{4}$, contemplando o projeto pedagógico "centrado no aluno como sujeito da aprendizagem e apoiado no professor como facilitador e mediador do processo ensino-aprendizagem" ${ }^{11}$. Em nossa instituição, ocorreram várias atividades, como simpósios e grupos de trabalho durante o ano letivo de 2004, com a participação discente e docente. Questionamentos levantados durante essas atividades motivaram a realização desta pesquisa, visto que, para criar ou aperfeiçoar mecanismos de aproveitamento de conhecimentos, a avaliação da qualidade do ensino pode servir como parâmetro de retroalimentação da eficiência do mesmo. Assim, este trabalho tem como objetivo conhecer o estado atual e os métodos propostos sob o ponto de vista dos estudantes para facilitar a aprendizagem da Semiologia Médica e comparar estas informações com as disponíveis na literatura.

\section{METODOLOGIA}

Trata-se de um estudo descritivo observacional e de caráter transversal. Dele participaram 159 alunos da Faculdade de Medicina da Universidade Federal de Minas Gerais (UFMG) matriculados no primeiro semestre de 2004 e que haviam cumprido a disciplina de Semiologia Médica de adultos. Esta disciplina é ministrada durante dois semestres letivos, com uma carga horária total de 435 horas, englobando a semiologia de crianças e adolescentes, adultos e a semiologia de algumas especialidades (dermatologia, aparelho locomotor, otorrinolaringologia, oftalmologia e neurologia). A semiologia de adultos é ministrada com uma carga didática de 60 horas, referentes à Semiologia I, e de 126 horas, referentes à Semiologia II. O objetivo principal do ensino da Semiologia I é a realização com proficiência da entrevista médica e do exame físico geral, introduzindo o aluno no conhecimento do método clínico. O cenário de prática dessa disciplina é o ambulatório Bias Fortes do Hospital das Clínicas da UFMG. Durante o ensino da Semiologia II, o conteúdo programático teórico e prático inclui a abordagem de pacientes com sintomas e sinais específicos dos aparelhos cardiovascular, respiratório e digestivo, com ênfase no exame físicoespecífico, sendo também objetivo a discussão em termos fisiopatológicos e psicodinâmicos para se estabelecer o diagnóstico sindrômico. Essa disciplina é ministrada principalmente nas enfermarias do Hospital das Clínicas, em sistema de rodízio. As turmas são compostas por seis a sete alunos por professor. A parte teórica, que corresponde a $25 \%$ da carga horária total, é ministrada por esse mesmo professor sob a forma de grupos de discussão do conteúdo programático preestabelecido.

A participação dos alunos foi voluntária e anônima, por meio do preenchimento de um questionário, especificamente elaborado para esta pesquisa e administrado no último dia letivo. O questionário foi composto de cinco partes: a) dados dos alunos (idade e sexo); b) importância das disciplinas ministradas no atual ciclo básico para o ensino-aprendizagem em Semiologia Médica de adultos; c) avaliação da metodologia atual empregada nessa disciplina; d) auto-avaliação quanto aos objetivos gerais da disciplina; e) propostas de mudança.

O questionário foi composto para avaliar o ensino da Semiologia de Adultos de responsabilidade do Departamento de Clínica Médica e considerando-se as discussões ocorridas durante seminários e reuniões do projeto de Mudança Curricular ${ }^{5}$. Foram avaliadas as seguintes disciplinas do atual ciclo básico com interface direta no ciclo propedêutico: anatomia médica, fisiologia médica, embriologia, química fisiológica, bioquímica e citologia/ histologia. A cada uma foi atribuída uma pontuação entre 1 e 5 , na qual 1 representa pouco importante, e 5, muito importante. A avaliação da metodologia atual do ensino dessa disciplina englobou o cenário de prática, a relação numérica aluno/ professor, o número de pacientes atendidos, a infra-estrutura, o sistema de rodízio, a discussão dos casos clínicos, a parte teórica e a carga horária. Na auto-avaliação, as habilidades para realizar a anamnese, o exame físico do paciente e o raciocínio fisiopatológico foram os itens questionados. Quanto às propostas para mudança no cenário de prática, foi feita uma pergunta que oferecia ao aluno três opções (somente no ambulatório, somente na enfermaria ou em ambos os cenários) para uma aprendizagem mais adequada e foi solicitada a justificativa para sua resposta. Também foram feitas perguntas abertas sobre sugestões de aspectos que conservaria e aspectos que modificaria no curso atual. 
Para a análise dos dados, foi utilizado o programa SPSS. Os resultados foram expressos em números e proporção quando se tratava de variáveis discretas, e em medidas de tendência central (média) para as variáveis contínuas. As respostas às perguntas abertas foram analisadas de acordo com as proporções das sugestões mais freqüentes.

\section{RESULTADOS}

Participaram do estudo 157 alunos (98,7\%). A idade média dos estudantes foi de 22,4 $\pm 1,4$ anos, variando entre 20 e 29 anos. A maioria era do sexo masculino (85 alunos, $54,1 \%$ ). A importância das disciplinas ministradas no atual ciclo básico para o aprendizado da Semiologia foi a seguinte, em ordem decrescente, segundo a média da pontuação de cinco graduações: fisiologia $(4,7 \pm 0,6)$, anatomia $(4,2 \pm 1,0)$, química fisiológica $(3,4 \pm 1,1)$, citologia/histologia $(2,8 \pm 1,2)$, bioquímica $(2,1 \pm 1,1)$ e, por último, embriologia $(2,0 \pm 1,0)$.

O cenário atual de prática referente aos módulos do sistema digestivo e do sistema cardiovascular foi a enfermaria para $75,2 \%$ e $84,7 \%$ dos alunos, respectivamente. O ensino do sistema respiratório foi ministrado para 36,9\% dos alunos no ambulatório, para 47,8\% nas enfermarias e para $12,7 \%$ dos alunos em ambos os ambientes (Tabela 1).

TABELA 1

\section{Cenário atual de prática}

\begin{tabular}{llll}
\hline Módulos/Prática & \multicolumn{1}{c}{ Aparelho respiratório } & Aparelho digestivo Aparelho cardiovascular \\
\hline Ambulatório & $36,9 \%$ & $12,1 \%$ & $2,5 \%$ \\
Enfermaria & $47,8 \%$ & $75,2 \%$ & $84,7 \%$ \\
Ambos & $12,7 \%$ & $6,4 \%$ & $6,4 \%$ \\
Não definido & $2,5 \%$ & $6,4 \%$ & $6,4 \%$ \\
\hline
\end{tabular}

Noventa alunos $(57,3 \%)$ consideraram adequada a relação numérica entre alunos e professor, de $7 / 1$. Os demais consideraram que a melhor relação seria de $4 / 1$ ou $5 / 1$. Cento e dezessete alunos $(74,5 \%)$ consideraram insuficiente o número de pacientes examinados.

A infra-estrutura foi considerada inadequada por $60,5 \%$, sendo apontada como justificativa a falta de materiais para a semiotécnica específica, como otoscópio e oftalmoscópio, e a falta de salas específicas para o ensino próximas às enfermarias. A carga horária foi considerada pequena ou pouco aproveitada por 88 alunos (56,1\%).

A maioria dos alunos (54,1\%) considerou-se apta a realizar de maneira satisfatória a anamnese. Uma proporção menor
$(22,9 \%)$ referiu apresentar dificuldade para ordenar os dados, e $12,1 \%$ referiram que entrevista parcialmente os pacientes. Cento e dois estudantes $(65 \%)$ se consideraram aptos para examinar o paciente, porém sem a habilidade necessária, e $18,5 \%$ referiram não perceber sinais importantes ao exame físico (Tabela 2).

Quando questionados sobre a aptidão para realizar o raciocínio fisiopatológico dos sinais e sintomas, 95 alunos (60,5\%) não se consideraram aptos. A maioria daqueles que justificou a resposta $(80,8 \%)$ apontou a falta, nesse nível do curso, de conhecimentos e experiência, e uma proporção relativa de $8,8 \%$ justificou sua inabilidade devido à fragmentação vertical com a disciplina de fisiologia, atualmente ministrada no ciclo básico.

TABELA 2

Habilidades dos estudantes segundo sua auto-avaliação

\begin{tabular}{lrlr}
\hline \multicolumn{2}{l}{ Habilidade para realizar a anamnese } & \multicolumn{2}{l}{ Habilidade para realizar o exame físico } \\
\hline Somente com o roteiro ao lado & $5,2 \%$ & Com ajuda do professor & $10,1 \%$ \\
De maneira parcial & $12,1 \%$ & Ainda sem habilidade & $65 \%$ \\
& & necessária & \\
Dificuldade em ordenar os & $28,6 \%$ & Sem perceber sinais & $18,5 \%$ \\
dados & & importantes & \\
De maneira adequada & $54,1 \%$ & De maneira adequada & $6,4 \%$ \\
\hline
\end{tabular}


As propostas de mudanças foram feitas por 139 alunos (88,5\%), predominando as propostas de aumento ou melhor aproveitamento da carga horária, reservando maior tempo para o rodízio e possibilitando uma assistência de maior número de pacientes e melhora da infra-estrutura. Foram propostos também a uniformização dos conteúdos e métodos de ensino, temas do conteúdo programático ministrados em aulas teóricas e composição do quadro docente específico para lecionar semiologiala (Tabela 3).
Apenas dois alunos sugeriram a realização de sessões clínicas (à semelhança das sessões clínico-radiológicas e anatomoclínicas dos internatos). Entre os pontos a conservar, foram citadas a pequena relação numérica entre alunos e professores, a autonomia dos alunos para realizar a anamnese e o exame físico, a relação profissional que o aluno já estabelece com o paciente, a dedicação de alguns professores, a metodologia da semiologia dos aparelhos em módulos e a teoria em grupos de discussão interativos.

TABELA 3

Propostas de mudanças sob o olhar do aluno

\begin{tabular}{ll}
\hline Propostas & $\begin{array}{l}\text { Percentual dos } \\
\text { respondedores }\end{array}$ \\
\hline $\begin{array}{l}\text { Aumento/melhor aproveitamento da carga horária } \\
\text { com maior assistência, melhora da infra -estrutura }\end{array}$ & \\
Uniformização do ensino & $18,7 \%$ \\
Seleção/composição do quadro docente específico & $16,5 \%$ \\
para Semiologia & \\
Conteúdo programático em aulas teóricas & $12,2 \%$ \\
\hline
\end{tabular}

Ainda em relação às propostas e quanto ao cenário de prática considerada mais adequada pelos acadêmicos para a aprendizagem de semiologia, a grande maioria (144 alunos, 91,7\%) assinalou a opção de ambos os cenários (no ambulatório e na enfermaria). A justificativa foi a riqueza de sintomas e sinais dos pacientes da enfermaria e o tempo mais disponível para realizar a anamnese daqueles pacientes do ambulatório, permitindo, dessa maneira, o contato com pacientes com quadros clínicos distintos. Apenas 5,7\% assinalaram como cenário mais adequado somente o ambulatório e 2,5\% assinalaram somente a enfermaria. A preferência daqueles pelo ambulatório foi justificada pela menor cooperação dos pacientes internados, pelo menor tempo de contato com eles devido à realização de exames complementares, resultando na ausência desses pacientes nos leitos, e pelo horário de visitas dos familiares.

\section{DISCUSS ÃO}

Com o objetivo de incentivar mudanças nos currículos das escolas médicas do Brasil para a formação de profissionais com perfil generalista e humanista, e capacitados para atuar em seus diferentes níveis de atenção com habilidades psicomotoras, cognitivas e afetivas, os ministérios da Saúde e Educação instituíram o Programa de Incentivo às Transformações Curriculares ${ }^{4}$. Foram incluídas 20 escolas nesse programa para desenvolverem experiências nucleares, capazes de determinar a integração universidade/serviço e nortear a formação médica contemplando a importância da participação do corpo discente. Dessa maneira, o modelo de ensino de transmissão, centrado na figura do professor, deve transformar-se no modelo pedagógico que privilegie "a participação ativa do aluno na construção do conhecimento e a integração entre os conteúdos", sendo o docente o facilitador desse processo de ensinoaprendizagem. Assim, o olhar do aluno sobre o estado atual do ensino e suas propostas permitem integrá-lo de maneira ativa nesse processo de transformação, no qual ele próprio é o sujeito da aprendizagem. Este trabalho reflete a motivação que o processo de transformação curricular de nossa faculdade ${ }^{5}$ desencadeou no corpo discente. Houve uma participação voluntária expressiva dos alunos que concluíam a disciplina de Semiologia. Sua percepção será valiosa no planejamento didáticopedagógico e como parte da avaliação da qualidade da docência, com implicações no programa curricular. 
A importância do ciclo básico para subsidiar a compreensão ampla dos problemas relacionados à saúde e à doença foi avaliada nesse trabalho. Em ordem decrescente, a fisiologia, a anatomia e a química fisiológica foram consideradas de relevância, devido aos seus conteúdos e a suas possibilidades de aplicação. Apesar disso, a maior parte dos alunos não se considerou apta a fazer o raciocínio fisiopatológico dos sinais e sintomas, e o justificou pela falta de conhecimentos e de integração com o ensino de fisiologia. Esta dicotomia entre o ciclo básico e o profissional do currículo atual é tema de debate há décadas. Por ocasião de um seminário promovido pela Associação Brasileira de Educação Médica em 1979, Sampaio 6 apontava alguns fatores que impediam a articulação entre esses ciclos, tais como a falta de integração vertical das disciplinas, a coordenação independente dos dois ciclos, a falta de interrelacionamento do pessoal docente e a distância física das áreas onde são lecionados os ciclos. Reconhece-se a importância dos conhecimentos do ciclo básico na formação, mas isso se torna evidente apenas durante o ciclo profissionalizante ${ }^{7}$, quando a aplicação clínica se faz presente. $\mathrm{O}$ volume de informações e detalhes, aliado à falta de conhecimento básico, por parte dos cientistas, dos mecanismos fisiopatológicos das doenças, contribui para essa desarticulação e não motiva a aprendizagem ${ }^{8}$. Essa lacuna entre ciências básicas e aplicações clínicas deve ser resolvida pela interdisciplinaridade, propiciando uma aprendizagem integrada com a convergência de várias áreas do conhecimento ${ }^{9}$.

Entre as disciplinas do ciclo básico, a embriologia foi considerada a de menor relevância para a prática médica pelo corpo discente. Essa disciplina propicia as bases para a compreensão da forma anatômica definitiva, para explicar determinadas associações clínicas e para o estudo das malformações. À semelhança das outras ciências básicas, a integração mais estreita com a anatomia morfológica e o estudo de quadros clínicos são necessários para realçar a utilidade da embriologia na resolução de problemas ${ }^{10}$, motivando o aluno para sua aprendizagem.

Apesar do avanço tecnológico na área médica, a pedra angular da medicina ainda é o método clínico. Esses recursos tecnológicos disponíveis poderão ser aplicados em sua plenitude somente quando se realizam uma anamnese e um exame físico com proficiência. A abordagem do paciente de maneira humanística e integral, estabelecendo uma relação médico-paciente adequada, possibilita a tomada de decisões, otimizando a aplicação dos recursos propedêuticos e evitando intervenções diagnósticas desnecessárias ou com eficácia e custo-efetividade duvidosos. E, como parte fundamental da assistência médica, a semiologia também tem sido, nos dias de hoje, validada por evidências científicas. Nesta era de sofisticada tecnologia, sintomas e sinais fornecem importantes informações sobre o diagnóstico e o prognóstico, norteando o manejo terapêutico dos pacientes ${ }^{11-13}$. Ainda assim, a habilidade para identificar sinais ao exame físico é pequena, inclusive quando se avaliam os egressos do curso de graduação de Medicina durante seu treinamento na residência ${ }^{14-16}$. Os educadores têm um papel crucial para realçar a importância da propedêutica clínica, despertando o interesse pelo método clínico durante o treinamento dos profissionais de saúde e motivando sua educação permanente.

No presente estudo, a visão do corpo discente quanto à infra-estrutura torna aparente a pequena valorização dada pela própria instituição ao ensino da semiologia. Outro fator influente é a profissionalização docente nos cursos de Medicina. Além da necessidade de capacitação docente, também apontada pelos alunos, outro desafio é o estímulo à docência. A criação de unidades de desenvolvimento educacional para a capacitação docente é necessária para implementar e consolidar currículos inovadores, mas ainda se encontra em estado embrionário nas instituições de ensino de saúde ${ }^{17}$. Por outro lado, os parâmetros mais utilizados para medir a produção docente são as publicações, permanecendo a docência como uma atividade marginal, incapaz de gerar recursos. Assim como são importantes as atividades de pesquisa e extensão, as atividades de ensino também fazem parte da excelência acadêmica, sendo necessários seu incentivo e recompensa.

Quanto ao desempenho dos estudantes, a maioria se considerou capaz de realizar a anamnese de maneira adequada ou com o roteiro ao lado, porém pouco menos de um terço relatou sua dificuldade em ordenar os dados. A maioria também referiu que realiza todo o exame físico, entretanto sem a habilidade necessária. Apesar das dificuldades no ensino da semiologia, em parte já apontadas, o aluno ainda fará sua formação no ciclo ambulatorial e de internatos, onde deve ter sua continuidade de estudos, aperfeiçoando a semiotécnica e o raciocínio clínico. A integração vertical ou a interdisciplinaridade são ferramentas importantes na abordagem pedagógica, auxiliando no processo de aquisição/construção do 
conhecimento, permitindo ao aluno internalizar e interpretar os conteúdos. Sua formação é um contínuo para adquirir competências e habilidades, devendo ser superado o conceito de disciplinas estanques, pois todas apresentam uma inter-relação dinâmica.

Nessa perspectiva, as estratégias didáticas devem favorecer a aprendizagem, valorizando o conhecimento prévio do aluno, a integração horizontal e vertical, e a aplicação das informações construídas pela busca ativa desse aluno. Apesar dessas diretrizes, o corpo discente ainda anseia pelo método tradicional, com a transmissão dos conteúdos pelo docente. Neste estudo, este anseio é refletido pelas propostas de uniformização do ensino e obtenção do conhecimento por meio de aulas teóricas. Um grande desafio da transformação curricular é construir coletivamente o projeto pedagógico centrado no aluno, fazendo com que ele acesse informações, formule hipóteses, fomente dúvidas, e seja o sujeito da aprendizagem com a ação facilitadora do docente. É um grande desafio não somente devido às dificuldades de capacitação docente já discutidas, como também devido ao comportamento passivo do aluno ${ }^{18-20}$. Por outro lado, a liberalização das atividades educacionais com a escolha dos conteúdos, métodos e sistema de avaliação por docentes com capacitação pedagógica inadequada cria mais distorções e heterogeneidade na formação discente, sendo incompatível com programas de excelência no ensino17. Assim, esses fatores devem ser levados em conta no planejamento curricular, com impactos na inserção docente e discente.

A preferência relatada pelos alunos neste estudo para o ensino-aprendizagem da semiologia pelo cenário de prática que inclua o ambulatório e a enfermaria é um aspecto de interesse e que tem sido tema de discussão na literatura pertinente. Observa-se um declínio do ensino à beira do leito, apesar de sua importância no treinamento discente $^{21}$. Devido às alterações na prática médica e na sociedade, vem ocorrendo uma mudança na assistência médica, com diminuição do tempo de permanência hospitalar dos pacientes e manejo em nível ambulatorial de muitas doenças que outrora eram diagnosticadas e tratadas por meio da internação dos pacientes ${ }^{22}$. Tudo isto, aliado às dificuldades do ensino à beira do leito - como sobrecarga de trabalho dos docentes com atividades administrativas e de pesquisa-assistência, constrangimento e/ou não-cooperação dos pacientes e ruído do ambiente , contribuiu para esse declínio ${ }^{23}$.

Há vantagens e desvantagens do ensino-aprendizagem em cada um desses cenários. A abordagem de condições agudas, com sintomas e sinais distintos, ensino sobre especialidades e maior treinamento em propedêutica e procedimentos são vantagens do ensino hospitalar. Por outro lado, habilidades de comunicação e aprendizagem sobre os aspectos psicossociais são competências que se adquirem principalmente no ambiente ambulatorial ${ }^{24-29}$. Assim, é necessária a integração curricular desses cenários de prática para que se proporcione ao corpo discente um método de ensino-aprendizagem mais efetivo para a aquisição de competências e habilidades gerais e específicas. Como métodos secundários de ensino, o uso de um laboratório de simulação com manequins e multimídia pode facilitar a aprendizagem e ser um estímulo para o desenvolvimento da autonomia do aluno ${ }^{30-33}$.

\section{CONSIDERAÇÕES FINAIS}

A transformação curricular a partir de objetivos educacionais para preparar o egresso do curso de graduação em Medicina para o exercício de competências e habilidades para promoção, prevenção, recuperação e reabilitação à saúde implica grandes desafios. Essa construção didático-pedagógica deve ser legítima, transpondo os vieses políticos e ideológicos e identificando as reais necessidades dessa transformação.

Neste sentido, é oportuna a análise da visão discente do estado atual do ensino e de suas propostas. Essa integração do aluno proporciona subsídios para o planejamento curricular e o estimula na participação ativa dessa construção, cujo método de ensino-aprendizagem deve ser centrado nesse próprio estudante.

\section{REFERÊNCIAS}

1. Brasil. Ministério da Educação. Conselho Nacional de Educação. Diretrizes Curriculares Nacionais do Curso de Graduação em Medicina (Brasília: Conselho Nacional de Educação/Câmara de Educação Superior, 2001), disponível em http://www.mec.gov.br/ sesu/diretriz.shtm

2. Comité Central, Instituto para la Educación Médica Internacional (IIME), New York, USA. Requisitos globales mínimos esenciales en educación médica. Educación Médica 2003; 6 (supl 2):11-19.

3. WFME Task Force on Defining International Standards in Basic Medical Education. Report of the Working Party, Copenhagen, 14-16 october 1999. Med. Educ 2000; 34(8):665-75. 
4. Ministério da Saúde. Secretaria de Políticas de Saúde. Uma nova escola médica para um novo sistema de saúde: Saúde e Educação lançam programa para mudar o currículo de medicina. Rev. Saúde Públ 2002; 36 (3):375-78.

5. Faculdade de Medicina da Universidade Federal de Minas Gerais. Projeto de Mudança Curricular do Curso Médico, 2002.

6. Sampaio ER. Relatório final do tema: "articulação ciclo básico/ciclo profissional”. Rev. Brás. Educ. Méd 1979; 3 (3):93-4.

7. Lobo LCG. Ensino das matérias básicas. Rev. Brás. Educ. Méd 1981; 5 (2):95-105.

8. Arias I M. Training basic scientists to bridge the gap between basic science and its aplication to human disease. N Engl J Med 1989; 321 (14):972-74.

9. Marcondes E. Integração horizontal e vertical: os conjuntos de disciplinas na graduação médica. In: Marcondes E \& Gonçalves L. Educação médica. São Paulo, Sarvier, 1998 p.115-129.

10. Mompeó B y Pérez L. Relevancia de la anatomía humana en el ejercicio de la medicina de asistencia primaria y en el estudio de las asignaturas de segundo ciclo de la licenciatura en medicina. Educación Médica 2003; 6 (1):41-51.

11. Saunder G. The importance of the history in the medical clinic and the cost of unnecessary test. Am. Heart J. 1980; 100:928-31.

12. Drazner MH, RameJ E, Stevenson L W, Dries DL. Prognostic importance of elevated jugular venous pressure and a third heart sound in patients with heart failure. N. Engl. J. Med. 2001; 345 (8):574-81.

13. Relly BM. Physical examination in the care of medical inpatients: an observational study. Lancet 2003; 362:1100-5.

14. Etchells E, Bell C, Robb K. Does this patient have an abnormal systolic murmur? JAMA 1997; 277 (7):564-71.

15. Mangione S, Nieman LZ. Cardiac auscultatory skills of internal medicine and family practice trainees. A comparison of diagnostic proficiency. JAMA 1997; 278 (9):717-22.

16. Mangione S. Cardiac auscultatory skills of physiciansin-training: a comparison os three English-speaking countries. Am. J. Med 2001; 10:223-5.

17. Gordam PA. Currículos inovadores: o desafio da inserção docente. In: Batista NA, Batista SHSS, org.
Docência em saúde: temas e experiências. São Paulo, Editora Senac, 2004, p. 187-200.

18. Schamroth AJ, Haines AP. Student assessment of clinical experience in general surgery. Med. Teach 1992; 14(4):355-62.

19. Modell HI. Preparing students to participate in an active learning environment. Am J Physiol 1996; 270:S69-77.

20. Dolan S, Mallot DB, Emery JA. Passive learning: a marker for the academically at risk. Med Teach 2002; 24 (6):648-9.

21. Ahmed M El-Bagir K. What is happening to bedside clinical teaching? Med Educ 2002; 36 (12):1185-8.

22. Towle A. Changes in health care and continuing medical education for the 21 st century. BMJ 1998; 316:301-304.

23. Nair B R, Coughlan JL, Hensley M J. Impediments to bedside teaching. Med Educ 1998; 32 (2):159-62.

24. O'Sullivan M, Martin J, Murray E. Students' perceptions of the relative advantages and disadvantages of community-based and hospitalbased teaching: a qualitative study. Med Educ 2000; $34(8): 648-55$.

25. Bryant P, Hartley S, Coppola S, Berlin A, Modell M, Murray E. Clinical exposure during clinical method attachments in general practice. Med Educ 2003; 37(9):790-3.

26. Murray E, Jolly B, Modell M. A comparison of the educational opportunities on junior medical attachments in general practice and in a teaching hospital: a questionnaire survey. Med Educ 1999; 33 (3):170-6.

27. Nair BR, Coughlan JL, Hensley MJ. Students and patients perspectives on bedside teaching. Med Educ 1997; 31(5):3416.

28. Dolmans DHJM, Wolfhagen HAP, Essed G G M, Scherpbier AJJA, Van der Vleuten CPM. Students' perceptions of relationships between some educational variables in the out-patient setting. Med Educ 2002; 36(8):735-41.

29. Bligh J. Improving student learning. Med Educ 2002; 36(8):692.

30. Paul S, Dawson KP, Lanphear JH, Cheema M Y. Vídeo recording feedback: a feasible and effective approach to teaching history-taking and physical examination skills in undergraduate paediatric medicine. Med Educ 1998; 32(3):332-6. 
31. Finley JP, Sharratt GP, Nanton MA, Chen RP, Roy DL, Paterson G. Auscultation of the heart: a trialof cassroom teaching versus computer-based independent learning. Med Educ 1998; 32(4):357-61.

32. Brueckner JK, Traurig H. Students' responses to the introduction of a digital laboratory guide in medical neuroscience. Med Teach 2003; 25(6):643-8.

33. Jevtic J, Sebastian JL, Bragg D. Use of computer simulated lung sounds in a introductory to physical exam course: Do students think it works? J Gen Intern Med 2004; 19:98.

\section{CONFLITOS DE INTERESSE}

Declarou não haver

\section{ENDEREÇO PARA CORRESPONDÊNCIA}

Departamento de Clínica Médica da Faculdade de Medicina da UFMG

Avenida Alfredo Balena, 190 - sala 4070

30130-100 - Belo Horizonte - MG

E-mail: rosesilv@medicina.ufmg.br 